\title{
LNMR Study on Microstructure Characteristics and Pore Size Distribution of High-Rank Coals with Different Bedding
}

\author{
Jiajia Liu $\mathbb{D},{ }^{1,2,3}$ Jianmin Hu $\mathbb{D},,^{1}$ Mengqi Shen $\mathbb{D}^{1},{ }^{1}$ Ming Yang $\mathbb{D},{ }^{1,2,3}$ and Yingxiang Fang $\mathbb{D i D}^{1}$ \\ ${ }^{1}$ Henan Polytechnic University, School of Safety Science and Engineering, Jiaozuo, Henan 454003, China \\ ${ }^{2}$ Henan Polytechnic University, Collaborative Innovation Center of Coal Work Safety and Clean High Efficiency Utilization, \\ Jiaozuo, Henan 454003, China \\ ${ }^{3}$ Henan Polytechnic University, Henan Provincial Key Laboratory of Gas Geology and Gas Control Provincial and \\ Ministry of State Key Laboratory Breeding Base, Jiaozuo, Henan 454003, China
}

Correspondence should be addressed to Jiajia Liu; liujiajia@hpu.edu.cn

Received 7 June 2021; Accepted 21 September 2021; Published 7 October 2021

Academic Editor: Peng Liu

Copyright $(2021$ Jiajia Liu et al. This is an open access article distributed under the Creative Commons Attribution License, which permits unrestricted use, distribution, and reproduction in any medium, provided the original work is properly cited.

In order to study the pore structure characteristics of high-rank coals with different bedding, NMR experiments were carried out for high-rank coals with different bedding angles $\left(0^{\circ}, 30^{\circ}, 45^{\circ}, 60^{\circ}\right.$, and $\left.90^{\circ}\right)$. The results show that the distribution of $T_{2}$ map of high-rank coal with different bedding is similar to some extent, showing a double peak or triple peak distribution, and the first peak accounts for more than $97 \%$ of the total, indicating that small holes are developed in high-rank coal with different bedding, while macropores are not developed. The influence of bedding angle on the fracture proportion is less than $0.3 \%$. Compared with the fracture proportion, the effect of bedding angle on the proportion of microhole, medium hole, and large hole is greater and presents a certain rule. There are certain differences in $T_{2}$ cutoff value $\left(T_{2 \mathrm{C}}\right)$ of high-rank coal with different bedding. The relationship between bedding angle and $T_{2 \mathrm{C}}$ conforms to exponential function, and the correlation degree $R^{2}$ is 0.839 . The research results provide a theoretical basis for gas extraction and utilization and prevention of gas disaster in coal mines in China.

\section{Introduction}

Coal is the main source of energy in China and plays an important role in China's economic development. China is rich in coal and coalbed methane reserves, but the conditions of coal reservoirs are complex. The high gas mines account for more than $65 \%$, and the total gas volume is 36 trillion $\mathrm{m}^{3}$. China is rich in high-rank coalbed methane resources, accounting for $1 / 3$ of the total high-rank coalbed methane resources. Coalbed methane mainly exists in the coal seam in the state of adsorption. The exploitation of coalbed methane is a continuous process of desorption-diffusion-seepage, which is carried out in the pores and cracks of coal body. Coal has typical anisotropic characteristics in different bedding and joint directions and is a medium with complex pore structure and multiscale structure [1]. Therefore, studying the structural characteristics of pores and fissures of different bedding high-rank coal samples has important guiding significance for studying the process of gas "desorptiondiffusion-seepage" and provides a certain theoretical basis for the extraction and utilization of coal mine gas and the prevention of gas disasters in our country. There are observation methods (such as transmission electron microscopy observation method and scanning electron microscopy observation method) and experimental testing methods (such as cryogenic liquid nitrogen test method, mercury intrusion method, and nuclear magnetic resonance test method) to study the pore structure of coal seams. Among them, the mercury intrusion method can test the pore and macropore structure of the sample, but it will cause irreversible damage to samples [2], while the nuclear magnetic resonance method has advantages of convenient detection and no damage to the test samples.

At present, many scholars at home and abroad have carried out research on the pore structure of coals. Some of 
them have conducted research using the mercury intrusion method, nitrogen adsorption method, or low-temperature $\mathrm{CO}_{2}$ adsorption method: Chen et al. [3] studied the pore structure and fractal characteristics of outburst coal by using the low-temperature liquid nitrogen adsorption test; Qin et al. [4] studied the pore structure characteristics of lignite, bituminous coal, and anthracite by using nitrogen adsorption and mercury intrusion methods; Li et al. [5] studied the pore structure characteristics of low coal ranks in the southern margin of Junggar Basin by using mercury intrusion method; Zhao et al. [6] used the mercury intrusion method to analyze in detail the complex characteristics of the pore structure of structural coal in Qinglong Coal Mine; Lin et al. [7] used nitrogen adsorption and mercury intrusion methods to analyze the pore structure characteristics of middle- and low-rank coals; Zhao et al. [8] comprehensively used high-pressure mercury injection, low-temperature liquid nitrogen adsorption, and lowtemperature $\mathrm{CO}_{2}$ adsorption experimental methods to conduct full-scale pore structure characterization studies on high-rank coals in Qinnan area and concluded that the pores of high-rank coals at all levels are relatively developed; Labani and Wang et al. [9, 10] used mercury intrusion and nitrogen adsorption methods to analyze the internal characteristics of the pore structure of the sample; and Fu et al. [11] used the nitrogen adsorption method to determine the basic characteristic parameters (such as pore volume and pore size distribution). Other researchers have used NMR methods to study: Yao et al. [12] reported a new method that applied low-field nuclear magnetic resonance technology and microfocus CT scanning technology to carry out fine quantitative characterization of coal pore and fissure types, effective porosity, pore structure distribution, and spatial configuration of pore and fissure; Wang et al. [13] used the nuclear magnetic resonance experiment method to analyze the characteristics of coal pore diameter before and after cold leaching in liquid nitrogen; Zheng et al. [14] analyzed the characteristics of movable fluid, bound fluid, and pore size distribution in Huainan lowrank coal reservoir by NMR experiment and concluded that adsorption pore is the most developed in low-rank coal in Huainan, followed by seepage pore and fracture; Xie et al. [15] used low-temperature liquid nitrogen adsorption experiment and low-field NMR experiment to analyze the pore size distribution characteristics of middle-rank coals and high-rank coals and obtained the conversion coefficient $C$ between the relaxation time of NMR $T_{2}$ and the pore size distribution of coal micropores; Yang and Liu [16] used low-field NMR experiments to study the pore development characteristics of high-rank coal and concluded that fractures in high-rank coal did not develop, while micropores and small pores are developed; Liu et al. [17] used low-field NMR experiments to explore the reservoir structure characteristics of high-rank coals and concluded that high-rank coals in the direction of parallel bedding and vertical bedding have similar pore size distribution characteristics; Ren et al. [18] studied the characteristics of coal pore size and effective porosity of low-rank coal by lowfield NMR experiment; Xu et al. [19] studied the pore distribution characteristics of coal samples in Pingmei No. 10 Mine by low-field NMR technology; Yao and Liu [20], based on the low-field NMR system experimental analysis of the Longmaxi Formation marine shale in Sichuan Basin, proposed a relatively complete set of fine quantitative characterization technology for shale porosity, permeability, pore type, pore structure, and methane adsorption capacity; Yao et al. [21] used an NMR pore structure model to effectively estimate the pore size distribution of coals; Zhou et al. [22] used the low-field NMR method to study the relationship between fractal analysis and physical properties of pore fractures with low-rank coal; Cai et al. [23] used the nuclear magnetic resonance technology to study parallel bedding coal samples and found that the distribution of nuclear magnetic resonance transverse relaxation saturated water cores has a strong relationship with the pore structure and rank of coals; and Li et al. [24] used the nuclear magnetic resonance technology to study the pore distribution of coals with different structures.

At present, domestic and foreign scholars have conducted relevant research studies on the pore structure and characteristics of low-, medium-, and high-rank coals. However, there are few studies on the pore structure of high-rank coals of different bedding through low-field nuclear magnetic resonance technology. Furthermore, high-rank coals and coalbed methane resources are rich in our country, about a third of the total coalbed methane resources; therefore, it is of great significance to study the pore structure of high-rank coal with different bedding. Because of the advantages of low-field NMR technology, such as fast detection speed and no damage to test samples, the author collected high-rank coal from Zhongmacun Mine of the coking coal group and used NMR technology to test the pore structure characteristics of high-rank coal with different bedding angles $\left(0^{\circ}, 30^{\circ}, 45^{\circ}, 60^{\circ}\right.$, and $\left.90^{\circ}\right)$. The research results provide a theoretical basis for gas extraction and utilization and prevention of gas disaster in coal mines in China.

\section{Basic Theory of NMR}

NMR is the response of an atomic nucleus to radio frequency magnetization by a magnetic field. Many nuclei have a net magnetic moment and angular momentum (or spin). When there is an external magnetic field, the nucleus moves around the direction of the external magnetic field. When the magnetic nuclei of these spins interact with an external magnetic field, a measurable signal can be generated. If one or both of the number of neutrons and the number of protons in the nucleus are odd numbers, the conditions for generating nuclear magnetic resonance signals are available, such as hydrogen nucleus $1 \mathrm{H}$, carbon $13 \mathrm{C}$, and nitrogen $14 \mathrm{~N}$. Because hydrogen nuclei $1 \mathrm{H}$ are abundant in nature and easy to detect, almost all nuclear magnetic resonance techniques are based on the response of hydrogen nuclei. For the fluid in rock pores, there are three different relaxation mechanisms: free relaxation, surface relaxation, and diffusion relaxation [25]. The transverse relaxation time can be expressed as follows: 


$$
\frac{1}{T_{2}}=\frac{1}{T_{2 \mathrm{~B}}}+\frac{1}{T_{2 \mathrm{~S}}}+\frac{1}{T_{2 \mathrm{D}}},
$$

where $T_{2 \mathrm{~B}}$ is the free relaxation time, $T_{2 \mathrm{~S}}$ is the surface relaxation time, and $T_{2 \mathrm{D}}$ is the diffusion relaxation time [26]. Free relaxation is the inherent relaxation property of liquids, which is determined by the physical properties of liquids (such as viscosity and chemical composition). The surface relaxation strength varies with the change of rock properties. As with free relaxation, physical properties (such as viscosity and molecules) constitute the control of diffusion coefficients; in addition, environmental conditions, temperature, and pressure also influence diffusion.

Since the experimental environment uses a uniform magnetic field, based on the principle of nuclear magnetic resonance [27], equation (1) can be written as follows:

$$
\frac{1}{T_{2}}=\rho_{2}\left(\frac{S}{V}\right)
$$

where $S$ is the surface area of the pores, $\mu \mathrm{m}^{2} ; V$ is the pore volume [28], $\mu \mathrm{m}^{3}$; and $\rho^{2}$ is the transverse surface relaxation strength of the rock, $\mu \mathrm{m} / \mathrm{ms}$, with an element of $\mathrm{m} / \mathrm{ms}$. According to equation (2), the relaxation time $T_{2}$ is proportional to the pore radius $r$ :

$$
r=C T_{2},
$$

where $r$ is the pore radius, $\mathrm{nm}$; $C$ is the conversion coefficient $[29,30]$; and $T_{2}$ is the transverse relaxation time.

\section{Experimental Method and Process}

3.1. Laboratory Equipment. In this experiment, the MesoMR23-060H-I low-field NMR system of Suzhou Niumai Technology was used (the resonance frequency of the device was $21.67568 \mathrm{MHz}$, the magnetic field intensity was $0.5 \mathrm{~T}$, the magnet temperature was constant at $32 \pm 0.01^{\circ} \mathrm{C}$, and the RF pulse frequency was $21.67568 \mathrm{MHz}$ ). The $2 \mathrm{XZ}-4 \mathrm{~B}$ vacuum saturation device and TGL-21M table top high speed refrigerated centrifuge were used. CPMG parameter settings of low-field NMR are shown in Table 1.

The physical map of the experimental test equipment is shown in Figure 1.

\subsection{Coal Sample Preparation and Experimental Procedures.} Collect fresh coal from Zhongmacun Mine of the coking coal group, and drill $\Phi 25 \mathrm{~mm} \times 50 \mathrm{~mm}$ cylindrical raw coal samples according to the angles of $0^{\circ}, 30^{\circ}, 45^{\circ}, 60^{\circ}$, and $90^{\circ}$ with the seam structure. These are ZM1, ZM2, ZM3, ZM4, and ZM5. The production process of the coal sample is shown in Figure 2.

The prepared coal pillars are reserved for the experiment, and the fresh small coal blocks that cannot be prepared into cylindrical coal blocks are tested for the maximum reflectance and microscopic components of the vitrinite coal. The test results are shown in Table 2.

Experiment with the coal samples ZM1, ZM2, ZM3, ZM4, and ZM5 prepared above: first, use the nuclear magnetic resonance system to test the $T_{2}$ pattern distribution
TABLE 1: CPMG sequence parameter.

\begin{tabular}{lcccccc}
\hline $\begin{array}{l}\text { Parameter } \\
\text { name }\end{array}$ & $\begin{array}{c}\text { SW } \\
(\mathrm{kHz})\end{array}$ & $\begin{array}{c}\text { TE } \\
(\mathrm{ms})\end{array}$ & $\begin{array}{c}\text { SF } \\
(\mathrm{MHz})\end{array}$ & $\begin{array}{c}\text { RFD } \\
(\mathrm{ms})\end{array}$ & $\begin{array}{c}\text { TW } \\
(\mathrm{ms})\end{array}$ & NECH \\
\hline Value setting & 250 & 0.251 & 21 & 0.08 & 5000 & 10000 \\
\hline
\end{tabular}

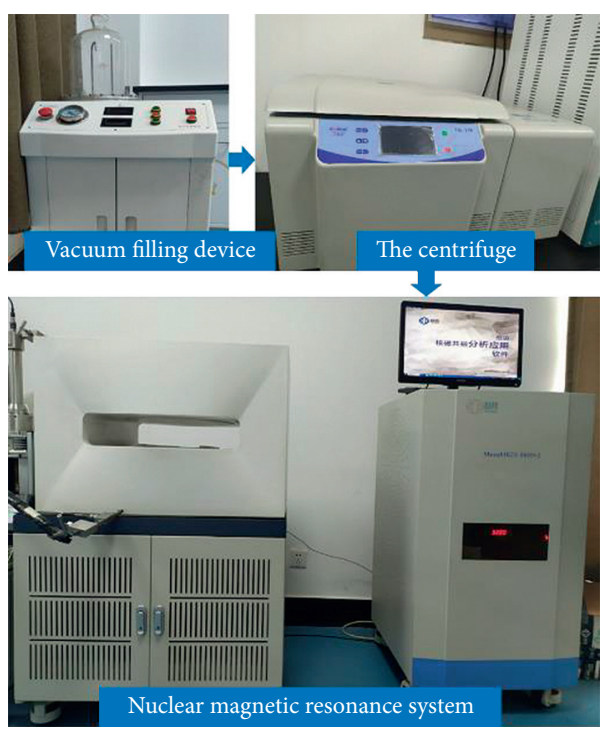

FIGURE 1: System diagram of experimental equipment.

of the natural state coal samples, put the coal samples ZM1, ZM2, ZM3, ZM4, and ZM5 into the vacuum saturation device which is saturated with water for 12 hours to fully saturate the coal sample (the quality difference between the two measured coal samples is less than $0.05 \%$ ), and then test the $T_{2}$ spectrum distribution of the saturated coal sample. The saturated coal samples were centrifuged for 0.5 hours at $1.38 \mathrm{MPa}$ centrifugal force, and the centrifuged coal samples were subjected to nuclear magnetic resonance tests [14]. The basic physical parameters of coal samples are shown in Table 3.

\section{Experimental Results and Discussion}

According to the experimental procedures, the low-field nuclear magnetic resonance system was used to perform nuclear magnetic resonance tests on ZM1, ZM2, ZM3, ZM4, and ZM5. The measured $T_{2}$ spectrum in the natural state is shown in Figure 3, the $T_{2}$ spectrum in the saturated state is shown in Figure 4, the $T_{2}$ spectrum of the coal sample after centrifugation is shown in Figure 5, and the histogram of the coal sample pore size distribution is shown in Figure 6.

Analyzing Figures 3-6, we can see that the $T_{2}$ spectrum of the coal sample has a double-peak or three-peak distribution. The $T_{2}$ spectrum of ZM4 has a three-peak distribution, and the $T_{2}$ spectrum of ZM1, ZM2, ZM3, and ZM5 has a double-peak distribution. The areas of the first peaks of the $T_{2}$ spectra of ZM3 and ZM5 are 40 999.437, 29 781.694, 25 264.928, 22 693.186, and 27 466.478, respectively, accounting for 98.61\%, 99.664\%, 99.524\%, 97.363\%, and $99.657 \%$, respectively; the areas of the second peak are $577.914,100.352,120.783,568.658$, and 99.657, respectively, 

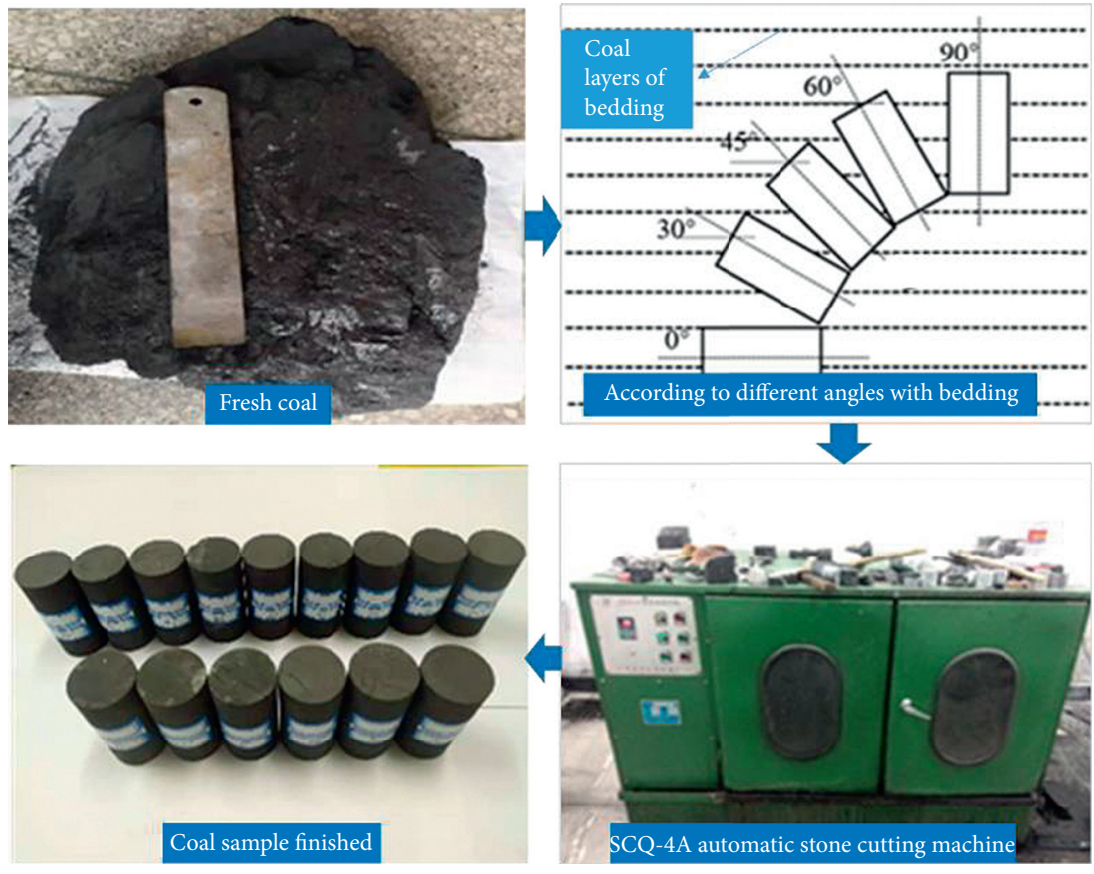

FIgURe 2: Production process drawing of coal samples.

TABLE 2: Microscopic coal and rock component detection results.

\begin{tabular}{lccccccccc}
\hline Results (\%) & Vitrinite & Inertinite & The total organic & Clay soil & Sulfide & Carbonate & $\begin{array}{c}\text { Amount } \\
\text { of inorganic }\end{array}$ & $\begin{array}{c}\text { Vitrinite average maximum } \\
\text { reflectivity }\left(R_{\text {max }}\right)\end{array}$ \\
\hline Value & 61.7 & 25.3 & 87.0 & 10.9 & 0.1 & 2.0 & 13.0 & 3.31 \\
\hline
\end{tabular}

TABLE 3: Basic physical property parameters of coal samples.

\begin{tabular}{|c|c|c|c|c|c|c|}
\hline Coal sample number & Height (mm) & Diameter $(\mathrm{mm})$ & Volume (ml) & $\begin{array}{c}\text { Natural state } \\
\text { quality (g) }\end{array}$ & $\begin{array}{l}\text { Saturated state } \\
\text { quality (g) }\end{array}$ & $\begin{array}{c}\text { Mass after } \\
\text { centrifugation }(\mathrm{g})\end{array}$ \\
\hline $\mathrm{ZM} 1\left(0^{\circ}\right)$ & 49.46 & 25.46 & 25.17 & 39.78 & 40.85 & 40.38 \\
\hline $\mathrm{ZM} 2\left(30^{\circ}\right)$ & 49.51 & 25.50 & 25.27 & 37.08 & 37.55 & 37.51 \\
\hline ZM3 $\left(45^{\circ}\right)$ & 49.55 & 25.48 & 25.25 & 35.80 & 36.25 & 36.19 \\
\hline $\mathrm{ZM} 4\left(60^{\circ}\right)$ & 49.57 & 25.51 & 25.32 & 37.85 & 38.16 & 38.10 \\
\hline ZM5 $\left(90^{\circ}\right)$ & 49.61 & 25.48 & 25.28 & 36.45 & 36.72 & 36.68 \\
\hline
\end{tabular}

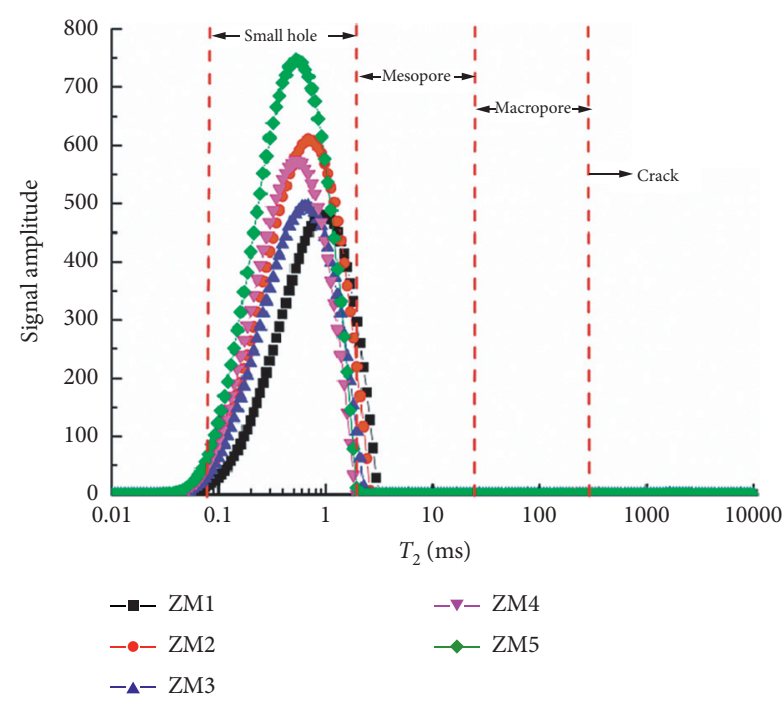

FIgURE 3: $T_{2}$ spectrum of coal samples in the natural state.

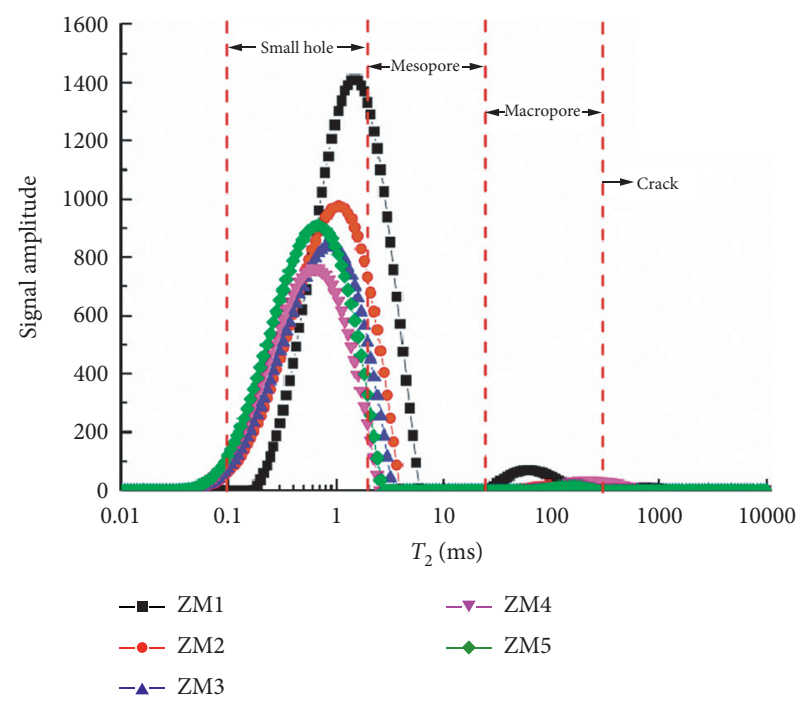

FIgURE 4: $T_{2}$ spectrum of coal samples in the saturated state. 


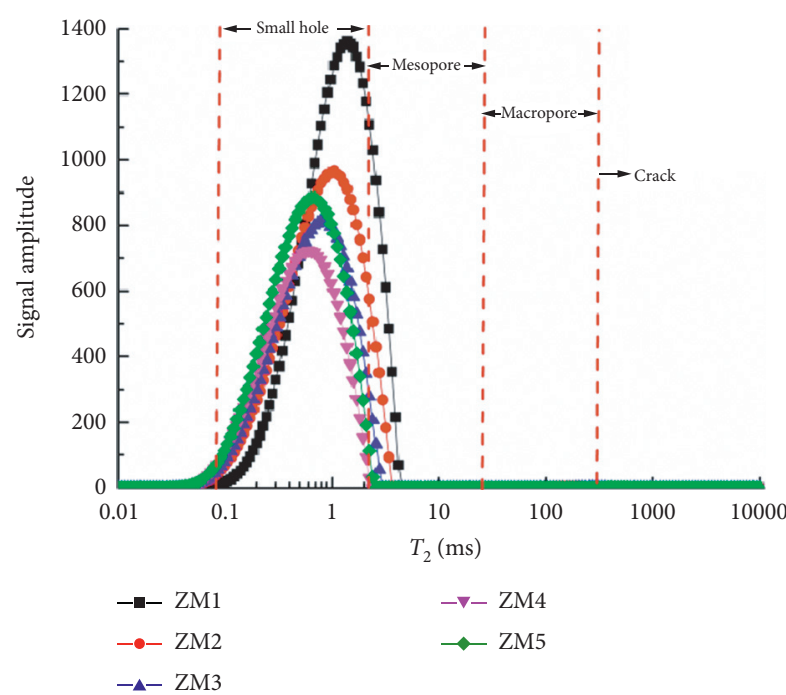

FIGURE 5: $T_{2}$ spectrum of coal samples after centrifugation.

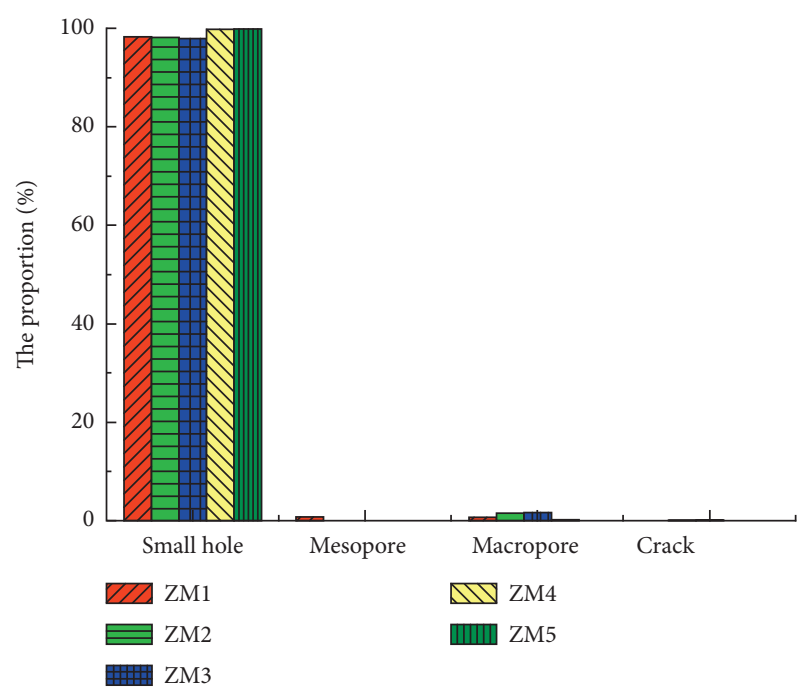

FIGURE 6: The histogram of coal pore size distribution.

and the proportions are $1.39 \%, 0.336 \%, 0.476 \%, 2.44 \%$, and $0.343 \%$, respectively; the area of the third peak of the $T_{2}$ spectrum of ZM4 is 45.95 , which accounts for $0.197 \%$; the proportion of the first peak of different bedding high-rank coals exceeds $97 \%$, of which the first peak of ZM2 has the highest proportion of $99.664 \%$, and the proportion of the first peak of ZM4 is the lowest, 97.363\%; among them, the second peak of ZM4 has the highest proportion of $2.44 \%$ and the second peak of ZM2 has the lowest proportion of $0.336 \%$. Further in-depth analysis shows that the $T_{2}$ pattern distribution of different bedding high-rank coals has certain similarities. The overall pattern shows that the proportion of small holes is relatively large, followed by the proportion of mesopores and macropores. This shows that high-rank coals with different bedding have developed small holes, while mesopores and macropores are not developed; the $T_{2}$ spectrum of ZM4 in the coal sample used in the experiment shows a three-peak distribution. The reason is that the difference in the bedding structure of the coal sample and the coal sample preparation process have caused some large pores to evolve into large cracks.

Figure 7 shows that the proportions of the pore diameters of different bedding high-rank coals are different. Among the proportions of micropores, ZM5 is the highest at $99.963 \%$ and ZM3 is the lowest at $97.977 \%$. This shows that, as the bedding angle increases, the proportion of micropores in coal samples first decreases and then increases; in the proportion of large holes, ZM3 is the highest at $1.726 \%$, and ZM5 is the lowest at $0.0006 \%$. As a whole, as the bedding angle increases, the proportion of large holes in coal samples first increases and then decreases; in the proportion of cracks, high-rank coals of different beddings show an " $\mathrm{N}$ " shape (first increase, then decrease, and then increase) distribution law, and the difference between the maximum and minimum proportions of cracks is less than $0.3 \%$. It shows that different bedding angles of high-rank coals have little effect on the ratio of cracks. The difference of the rational angle has little effect on the proportion of cracks. Compared with the proportion of cracks, the bedding angle has an influence of $1.886 \%, 0.885 \%$, and $1.725 \%$ on the proportions of micropores, mesopores, and macropores, respectively.

The $T_{2}$ cutoff value can be expressed as $T_{2 \mathrm{C}}$. The left side of $T_{2 \mathrm{C}}$ represents the bound fluid in the adsorption hole, and the right side represents the movable fluid in the percolation hole. We can calculate the bound fluid and movable fluid in the experimental sample through $T_{2 \mathrm{C}}$, the schematic diagram of $T_{2 \mathrm{C}}$ is shown in Figure 8. Draw a cumulative curve for the porosity components of the coal samples before and after centrifugation, and draw a straight line parallel to the time axis based on the maximum value of the cumulative porosity curve after centrifugation (indicated by the dotted line in the figure). This straight line is related to the saturated cumulative pores. The degree curve intersects at a point, denoted as point 1 , and then a straight line parallel to the time axis is made based on point 1 . This straight line intersects the time axis at point 2 , and the value of the time axis corresponding to point 2 is the desired value $T_{2 \mathrm{C}}$.

Analyzing Figures 8 and 9, we can see that $T_{2 \mathrm{C}}$ of different bedding high-rank coals has certain differences. According to the above method, $T_{2 \mathrm{C}}$ of different bedding high-rank coals ZM1, ZM2, ZM3, ZM4, and ZM5 can be obtained. They conform to the exponential function relationship; as shown in Figure 10, the function expression between them is $y=\exp (1.41647-0.02139 * x+1.35294 E-$ $4 * x^{2}$ ), and the correlation $R^{2}$ is 0.839 . It can be seen from Figure 10 that, as the bedding angle increases, $T_{2 \mathrm{C}}$ shows a trend of first decline and then rise. At the beginning, $T_{2 \mathrm{C}}$ decreases faster with the increase of the angle. When $T_{2 \mathrm{C}}$ drops to the lowest value, with the increase in bedding angle, $T_{2 \mathrm{C}}$ began to rise slowly.

Analyzing Figure 10, it can be seen that the water in the pores of high-rank coal with different bedding angles has different degrees of difficulty to be thrown out under the action of external centrifugal force. Based on the $T_{2 \mathrm{C}}$ calculation method described above, when the direction of the combined force of the centrifugal force and gravity on the water in the pores of the coal sample is closer to the bedding 


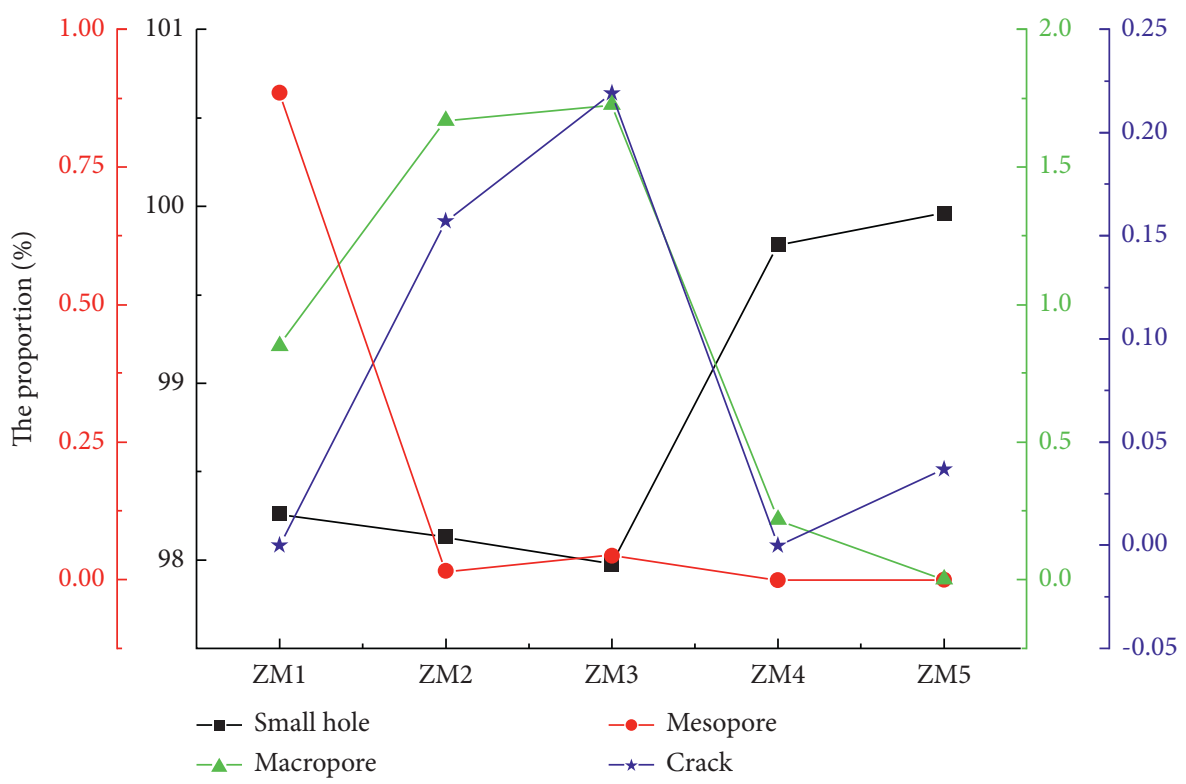

FIgURE 7: Pore proportion of high-rank coal with different bedding.

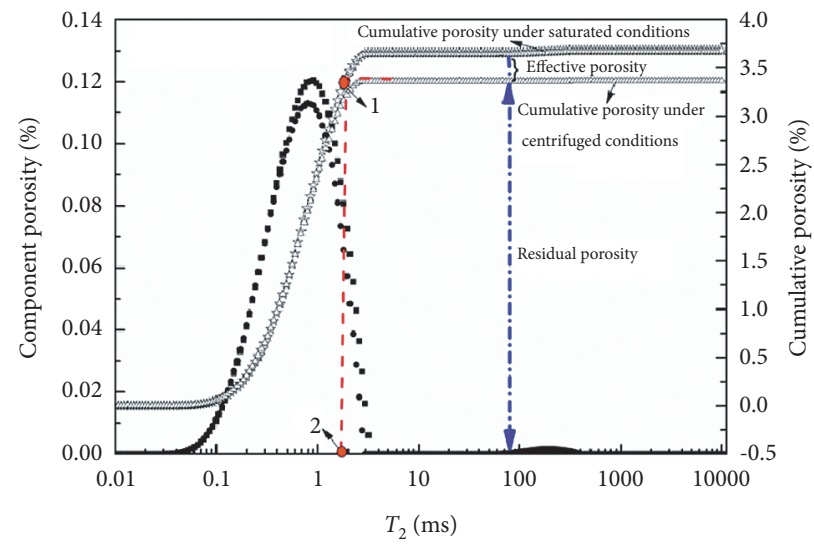

Saturated water porosity

- Centrifugal porosity

FIgURE 8: Schematic diagram of $T_{2 \mathrm{C}}$ of $\mathrm{ZM} 3$ coal sample.

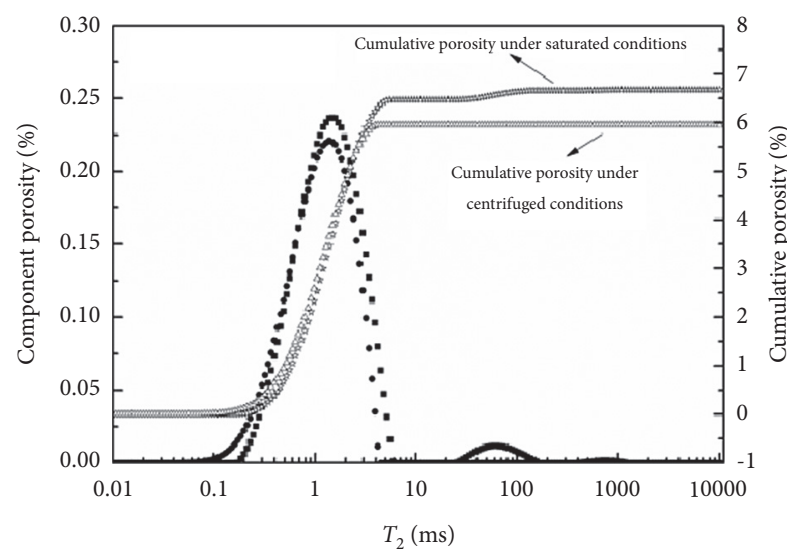

Saturated water porosity

Centrifugal porosity

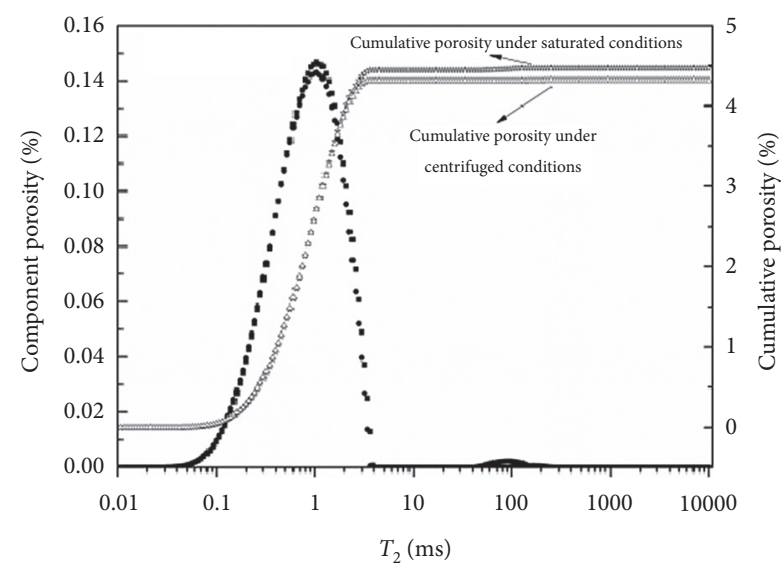

Saturated water porosity

Centrifugal porosity

Figure 9: Continued. 


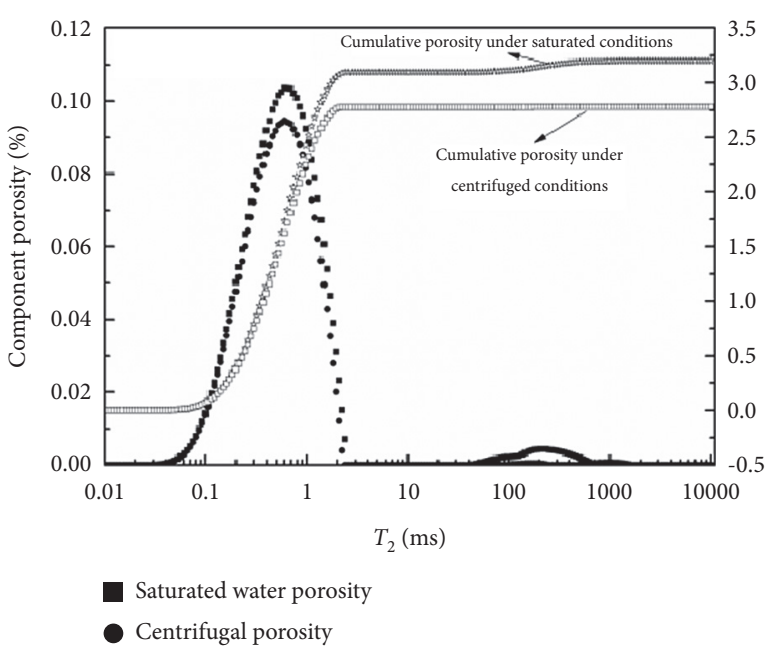

(c)

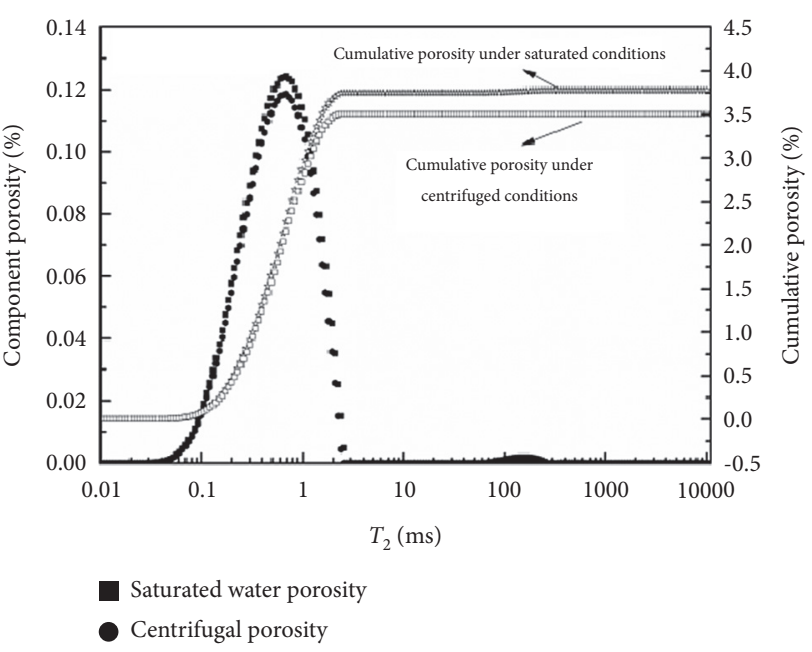

(d)

FIgURE 9: Porosity of high-rank coal with different bedding. (a) ZM1, (b) ZM2, (c) ZM4, and (d) ZM5.

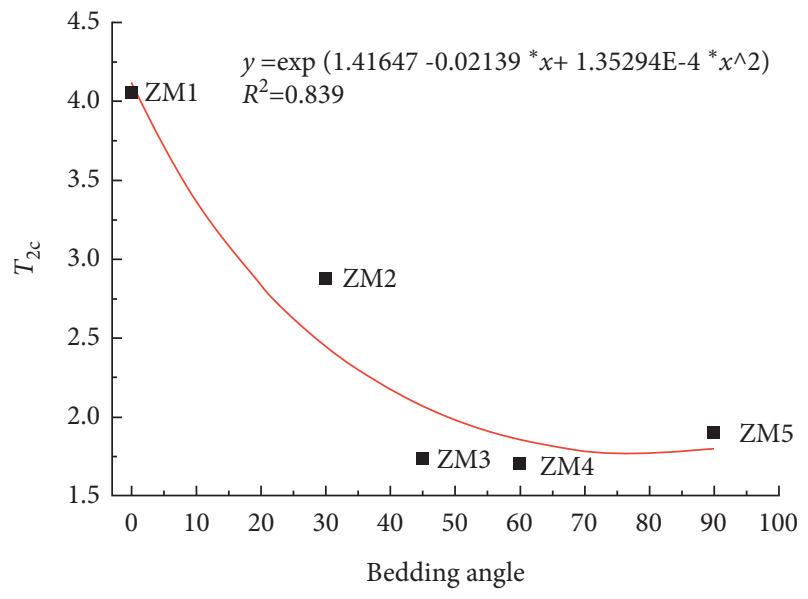

Figure 10: $T_{2 \mathrm{C}}$ of high-rank coal with different bedding.

direction of the coal samples, the water in the pores of the coal samples is easily thrown out; when the resultant direction of centrifugal force and gravity received by the water in the pore of the coal samples is inconsistent with the direction of bedding of the coal samples, the pore wall of the coal samples will give an opposite force to the water in the pore, and the more the resultant direction of centrifugal force and gravity deviates from the direction of bedding of the coal samples, the more difficult the water is to throw out.

\section{Conclusions}

(1) The $T_{2}$ patterns of different bedding high-rank coals have a certain regularity, showing a double-peak or triple-peak distribution. Their first peak accounts for a relatively high proportion, both exceeding $97 \%$. It is indicated that the micropores of different bedding high-rank coals are developed, while the medium and large pores are not developed.
(2) The different changing law of the angle is that the proportion of microholes first decreases and then increases with the increase of the bedding angle. The proportion of mesopores first rapidly decreases with the increase of the bedding angle and then basically remains unchanged. The proportion of large holes first increases and then decreases with the increase of the bedding angle.

(3) The bedding angle and $T_{2 C}$ conform to an exponential function relationship. $T_{2 C}$ shows a trend of first decline and then rise. At the beginning, $T_{2 C}$ decreases faster with the increase of the angle. When $T_{2 \mathrm{C}}$ drops to the lowest value, as the bedding angle increases, $T_{2 \mathrm{C}}$ shows a slow upward trend.

\section{Data Availability}

The data used to support the findings of this study are included within the article.

\section{Conflicts of Interest}

The authors declare that they have no conflicts of interest.

\section{Acknowledgments}

This work was supported by the National Natural Science Foundation of China (52074106 and 51734007). This project was also supported by Zhongyuan Postdoctoral Innovative Talent (ZYQR201810171), the China Postdoctoral Science Foundation (2019M652536), the Henan Postdoctoral Foundation (001801016), and the Doctoral Fund of Henan Polytechnic University (B2018-59).

\section{References}

[1] A. D. Alexeev, T. A. Vasilenko, and E. V. Ulyanova, "Closed porosity in fossil coals," Fuel, vol. 78, no. 6, pp. 635-638, 1999. 
[2] A. C. Patrick, J. R. Cathy, L. Esa et al., "Comparison of NMR cryoporometry, mercury intrusion porosimetry, and DSC thermoporosimetry in characterizing pore size distributions of compressed finely ground calcium carbonate structures," Industrial \& Engineering Chemistry Research, vol. 43, no. 43, p. 7920,2004

[3] L. Y. Chen, X. J. Li, Z. H. Shen, B. Juan, Y. Liu, and S. Xu, "Pore structure and fractal characteristics of outburst coal in northern Guizhou," China Safety Science Journal, vol. 30, no. 02 , pp. 66-72, 2020 .

[4] L. Qin, P. Wang, H. F. Lin, P. Zhao, C. Ma, and Y. Shi, "Advanced characterization of pore structure of liquid nitrogen frozen coal using nitrogen adsorption and mercury intrusion methods," Journal of Xi'an University of Science and Technology, vol. 40, no. 6, pp. 945-952, 2020.

[5] C. F. Li, Y. C. Wei, A. M. Wang, and D. Cao, "Low-ranked coal reservoir pore structure characterized by mercury intrusion-a case study of Southern Junggar basin," Coal Geology of China, vol. 30, no. 02, pp. 29-33, 2018.

[6] J. G. Zhao, M. Wang, and R. Y. Ma, Study on Pore Structure Characteristics of Qianxi Qinglong Coal Mine Based on Mercury Injection Method, pp. 1-11, Coal Science and Technology, Beijing, China, 2020.

[7] H. F. Lin, J. T. Bu, M. Yan, and Y. Bai, "Joint analysis of pore structure characteristics of middle and low rank coal with nitrogen adsorption and mercury intrusion method," Journal of Xi'an university of Science and Technology, vol. 39, no. 1, pp. 1-8, 2019.

[8] J. C. Zhao, Full Scale Pore Structure of High Rank Coals in the South of Qinshui basin and its Flow Difference, China University of Mining and Technology, Beijing, China, 2017.

[9] M. M. Labani, R. Rezaee, A. Saeedi, and A. A. Hinai, "Evaluation of pore size spectrum of gas shale reservoirs using low pressure nitrogen adsorption, gas expansion and mercury porosimetry: a case study from the Perth and Canning basins, western Australia," Journal of Petroleum Science and Engineering, vol. 112, no. 3, pp. 7-16, 2013.

[10] M. Wang and Q. Yu, "Pore structure characterization of Carboniferous shales from the eastern Qaidam basin, China: c," Journal of Petroleum Science and Engineering, vol. 152, pp. 91-103, 2017.

[11] H. Fu, X. Wang, L. Zhang et al., "Investigation of the factors that control the development of pore structure in lacustrine shale: a case study of block X in the Ordos basin, China," Journal of Natural Gas Science and Engineering, vol. 26, pp. 1422-1432, 2015.

[12] Y. Yao, D. Liu, Y. Cai, and J. Li, "Advanced characterization of pores and fractures in coals by nuclear magnetic resonance and X-ray computed tomography," Science China Earth Sciences, vol. 53, no. 6, pp. 854-862, 2010.

[13] C. X. Wang, X. B. Zhang, and F. C. Lu, "Study on coal cracking under liquid nitrogen soaking based on uclear magnetic resonance and stress analysis," China Safety Science Journal, vol. 29, no. 11, pp. 156-163, 2019.

[14] S. J. Zheng, Y. B. Yao, and Y. D. Cai, "Characteristics of dynamic fluid and pore diameter distribution of low-rank coal reservoirs in the southern margin of Junggar Basin," Coal Field Geology and Exploration, vol. 46, no. 1, pp. 56-60, 2008.

[15] S. B. Xie, Y. B. Yao, and J. Y. Chen, "Study on low-field nuclear magnetic resonance of micro-pore pore structure in coal reservoir," Acta Coal Sinica, vol. 40, no. S1, pp. 170-176, 2015.

[16] M. Yang and Y. P. Liu, "Experimental study on pore characteristics of high-order coal with low-field nuclear magnetic resonance," China science and technology of work safety, vol. 12, no. 11, pp. 63-69, 2016.

[17] S. Q. Liu, S. X. Sang, Y. H. Yang et al., "Structure features of high rank coal in parallel bedding and vertical bedding based on low field nuclear magnetic resonance," Coal Science and Technology, vol. 46, no. 10, pp. 110-116, 2018.

[18] H. K. Ren, A. M. Wang, C. F. Li, D. Cao, and Y. Wei, "Study on porosity characteristics of low-rank coal reservoirs based on nuclear magnetic resonance technology," Coal Science and Technology, vol. 45, no. 4, pp. 143-148, 2017.

[19] X. M. Xu, H. X. Ma, J. W. Tian, J. Wang, and G. Li, "Study on microscopic pore structures of coal based on nuclear magnetic resonance technology," Safety In Coal Mines, vol. 48, no. 2, pp. 1-4, 2017.

[20] Y. B. Yao and D. M. Liu, "Petrophysical properties and fluids transportation in gas shale: a NMR relaxation spectrum analysis method," Journal of China Coal Society, vol. 43, no. 1, pp. 181-189, 2018.

[21] Y. Yao, D. Liu, Y. Che, D. Tang, S. Tang, and W. Huang, "Petrophysical characterization of coals by low-field nuclear magnetic resonance (NMR)," Fuel, vol. 89, no. 7, pp. 1371-1380, 2010.

[22] S. Zhou, D. Liu, Y. Cai, and Y. Yao, "Fractal characterization of pore-fracture in low-rank coals using a low-field NMR relaxation method," Fuel, vol. 181, pp. 218-226, 2016.

[23] Y. Cai, D. Liu, Z. Pan, Y. Yao, J. Li, and Y. Qiu, "Petrophysical characterization of Chinese coal cores with heat treatment by nuclear magnetic resonance," Fuel, vol. 108, pp. 292-302, 2013.

[24] X. Li, Y. Kang, and M. Haghighi, "Investigation of pore size distributions of coals with different structures by nuclear magnetic resonance (NMR) and mercury intrusion porosimetry (MIP)," Measurement, vol. 116, pp. 122-128, 2017.

[25] Y. B. Yao and D. M. Liu, "Petrophysics and fluid properties characterizations of coalbed methane reservoir by using NMR relaxation time analysis," Coal Science and Technology, vol. 44, no. 6, pp. 14-22, 2016.

[26] A. Timur, "Nuclear magnetic resonance study of carbonates rocks," Log Analyst, vol. 13, no. 5, pp. 3-11, 1972.

[27] A. Rahila, F. Y. Ma, X. Zhang, J. Liu, M. Zhong, and X. Zhao, "Application of low-field nuclear magnetic resonance technology in coal petrographic pore structure," Nuclear Techniques, vol. 40, no. 12, pp. 43-48, 2017.

[28] Coates, R. George, L. Z. Xiao, and G. Prammer Manfred, NMR Logging Principles and Applications, Gulf Publishing Company, Houston, TX, USA, 1999.

[29] W. E. Kenyon, "Nuclear magnetic resonance as a petrophysical measurement," Nuclear Geophysics, vol. 6, no. 2, pp. 153-171, 1992.

[30] Y. Yao and D. Liu, "Comparison of low-field NMR and mercury intrusion porosimetry in characterizing pore size distributions of coals," Fuel, vol. 95, pp. 152-158, 2012. 